\title{
Swarm-Copters Senior Design Project: Simulating UAV Swarm Networks Using Quadcopters for Search and Rescue Applications
}

\author{
Author: Mark Moudy \\ Faculty Mentors: Kamesh Namuduri, Department of Electrical Engineering, College of \\ Engineering and David Keathly, Department of Computer Science and Engineering, College of \\ Engineering \\ Department and College Affiliation: Department of Computer Science and Engineering, \\ College of Engineering
}




\section{Bio:}

Mark Moudy is a senior undergraduate at the University of North Texas and will be graduating in May 2012 with a B.S. degree with a major in Computer Engineering and a minor in Mathematics. Mark has been an active member of IEEE and the IEEE Computer Society for the last two years. After graduation he will pursue a career in the Embedded and Real-Time Systems industry. 


\begin{abstract}
:
Currently when a person gets lost a rescue team is called into action to utilize a wide range of terrain specific methods and tools to help locate the missing person. This current method is problematic for several reasons, namely high costs and the increased risk of the rescuers becoming rescuees. Quadcopters are ideal for simulating a network of UAVs in a search and rescue situation because they can be outfitted with a range of situation specific sensors, are easily mobilized, and can reduce the risk to the rescuers. The Swarm-Copters system utilizes: multiple sensors to assist with flight, a camera system to provide a real-time view of the search area, and a centralized base station to control the search and rescue operation. The system was developed using a swarm style approach which allows for two or more autonomous quadcopters to work together in completing a scan of a search grid.
\end{abstract}




\section{Introduction}

The Swarm-Copters system was developed at the University of North Texas as part of the Department of Computer Science and Engineering Senior Design course during the 2011 - 2012 school year. The purpose of the Swarm-Copters system is to perform search and rescue operations by utilizing a swarm of autonomous UAVs to find missing persons while avoiding the risk of endangering lives and incurring exorbitant costs associated with traditional search and rescue (SAR) methods. The system was designed to utilize the Arducopter and QgroundControl open-source platforms.

\section{Background}

When a person goes missing, a rescue team is called into action to locate and rescue the missing person using a wide range of terrain-specific methods and tools. The goal of the SwarmCopters system is to lessen the impact of high SAR costs and secondary SAR operations for initial rescuer personal in distress. In 2005 the National Park Service executed a total of 2430 SAR operations at a cost of $\$ 4,996,705$ of which $\$ 2.1$ million was directly related to aircraft costs. [1] Refer to Table 1 for the cost breakdown.

\section{System Design}

The Swarm-Copters system is organized into two related but still independent subsystems, the quadcopters [Figure 1] and the base station [Figure 2]. The quadcopter sub-system is responsible for all flight functionality and receives task instructions from the base station which utilize the onboard sensors and flight features to execute SAR missions. The base station is responsible for mission planning, capturing in-flight data, and issuing instructions to the quadcopter swarm. These two sub-systems are tied together via an XBEE point-to-multipoint 
network that allows both sub-systems to pass messages through the MAVLink communications protocol. The relationship between the quadcopters and base station is illustrated in Figure 3.

\section{Functionality and Features}

The Swarm-Copters system is capable of performing a broad scan of a defined area in the event of an SAR by utilizing a swarm of UAVs to scan a search area and distribute the task among all available units. All UAVs checked into the system fly autonomously and are fitted with a range of sensors to provide real-time flight data and video feeds. UAVs are controlled via the base station which provides the operator with a heads up display of all flight information, mission planning and control capabilities. An automated flight planning widget has been implemented to generate all intermediate waypoints and distribute the current SAR tasks among all UAVs checked into the system.

Automated flight planning widget.

The Automated Flight Planning widget takes an input of either two or four control waypoints to define the boundaries of the search area. The widget automatically generates the UAV flight paths and evenly distributes the search area among all UAVs in the swarm through the use of a lawnmower search algorithm. [2] The automated flight plan also takes advantage of the evenly distributed workload among the UAVs to either

complete the search faster or expand the effective search range of the system. After the flight plan has been generated, the user has the option to save the flight plan, upload it to the quadcopters, or delete the flight plan and start over.

Search Algorithm. 
The Swarm-Copters system utilizes a lawnmower search algorithm to effectively traverse the search area and guarantee detailed coverage of the search area. [2] Each span lane generated for a given UAVs flight path is set to the center of the width of cameras field of view to ensure full coverage of the search area. The guiding principle for the implementation of this search algorithm is to read the current position of the UAV and then calculate the motion required to arrive at a target waypoint. Once motion is calculated we then calculate the heading trajectory and rate of speed reduction needed to stop the UAV at the ending waypoint.

Autonomous flight.

The UAV swarm is simulated with two or more quadcopters that are each independently capable of autonomous flight. The quadcopters receive messages from the base station containing waypoint information and the specific flight mode to use while traversing between waypoints. Currently the system uses five main flight modes during autonomous SAR execution. Refer to Table 2 for more information about flight modes.

\section{Hardware Components}

Each quadcopter is equipped with the following hardware components:

- Ardupilot Mega - Ardunio based UAV controller with Atmega2560

- Ardupilot Mega IMU Shield/ Oil pan with:

- Compass

- 3-Axis Gyroscope 
- Accelerometer

- Barometric Pressure Sensor - calculates altitude above ten feet

- Voltage Dividers - for monitoring battery levels

- $1.4 \mathrm{GHz}$ FPV Video System

- Ultrasonic Sensor - Calculate altitude under ten feet

- Mediatek GPS module

- XBEE-Pro 900MHz - Embedded RF module

- Three-Axis Magnetometer

\section{Important Legal Regulations}

\section{FAA Regulations}

A. Select an operating site that is of sufficient distance from populated areas. The selected site should be away from noise sensitive areas such as parks, schools, hospitals, churches, etc. [8]

B. Do not operate model aircraft in the presence of spectators until the aircraft is successfully flight tested and proven airworthy. [8]

C. Do not fly model aircraft higher than 400 feet above the surface. When flying aircraft within 3 miles of an airport, notify the airport, or when an air traffic facility is located at the airport, notify the control tower, or flight service station. [8]

D. Give right of way to, and avoid flying in the proximity of, full-scale aircraft. Use observers to help if possible. [8] 
E. Do not hesitate to ask for assistance from any airport traffic control tower or flight service station concerning compliance with these standards. [8]

\section{FCC regulations}

To operate video feeds on the $1.4 \mathrm{GHz}$ spectrum you will need a general class amateur radio license

\section{Future Plans}

The next iteration of the Swarm-Copters platform will focus heavily on object detection and avoidance. Currently, UAVs struggle with detection of obstacles on the ground and in the air. This issue requires an operator to closely monitor each UAV during flight and limits the effective range of flight during a mission. Once these issues are overcome, UAVs will become truly autonomous. 


\section{References}

[1] T. W. Heggie, M.E. Amundson, "Dead Men Walking: Search and Rescue in US National Parks," Wilderness and Environmental Medicine, Vol. 20, no. 3, pp. 244-249, 2009. ScienceDirect:http://www.sciencedirect.com/science/article/pii/S1080603209701280. [Accessed 04/12/2012]

[2] Worrall, Kevin J. "Guidance and Search Algorithms for Mobile Robots: Application and Analysis within the Context of Urban Search and Rescue." Sept. 2008. Web. 6 Apr. 2012. <http://theses.gla.ac.uk/508/01/2008worrallphd.pdf>. page 63

[3] Stable Mode, Arducopter: Arduino-based autopilot for multirotor craft, from quadcopters to traditional helis, [online] 19 Feburary 2012, http://code.google.com/p/arducopter/wiki /AC2_StableMode (Accessed: 12 April 2012).

[4] Auto Mode, Arducopter: Arduino-based autopilot for multirotor craft, from quadcopters to traditional helis, [online] 28 January 2012, http://code.google.com/p/arducopter/wiki /AC2_AutoMode (Accessed: 12 April 2012).

[5] Loiter Mode, Arducopter: Arduino-based autopilot for multirotor craft, from quadcopters to traditional helis, [online] 25 January 2012, http://code.google.com/p/arducopter/wiki /AC2_LoiterMode (Accessed: 12 April 2012).

[6] Simple Mode, Arducopter: Arduino-based autopilot for multirotor craft, from quadcopters to traditional helis, [online] 20 December 2011, http://code.google.com/p/arducopter/wiki /AC2_Simple (Accessed: 12 April 2012).

[7] Alt Hold, Arducopter: Arduino-based autopilot for multirotor craft, from quadcopters to traditional helis, [online] 19 Feburary 2012, http://code.google.com/p/arducopter/wiki /AC2_AltHoldMode (Accessed: 12 April 2012). 
[8] Model Aircraft Operating Standards, FAA Advisory Circular, AC 91-57, [online] 9 June 1981,http://rgl.faa.gov/Regulatory_and_Guidance_Library/rgAdvisoryCircular.nsf/0/1acf c3f689769a56862569e70077c9cc/\$FILE/ATTBJMAC/ac91-57.pdf (Accessed: 12 April 2012). 


\section{Tables and Figures}

Table 1: 2005 National Park Service Search and Rescue Costs [1]

\begin{tabular}{|l|r|}
\hline SAR Expense Category & Costs (2005) \\
\hline Personnel Costs & $\$ 2,300,000$ \\
\hline Aircraft Costs & $\$ 2,100,000$ \\
\hline Vessel Costs & $\$ 103,529$ \\
\hline Supply Costs & $\$ 469,361$ \\
\hline Total SAR Cost & $\mathbf{\$ 4 , 9 0 0 , 0 0 0}$ \\
\hline
\end{tabular}

Table 2: Flight Modes Used during UAV Flight

\begin{tabular}{|l|l|}
\hline Flight Mode & Description \\
\hline Stable & $\begin{array}{l}\text { Self-levels UAV, maintains current heading of } \\
\text { yaw, used for general flying [3] }\end{array}$ \\
\hline Auto & $\begin{array}{l}\text { UAV follows flight plan of waypoints along } \\
\text { with scripted take-off and landing } \\
\text { procedures[4] }\end{array}$ \\
\hline Loiter & $\begin{array}{l}\text { Attempts to maintain current location on yaw, } \\
\text { pitch and roll. [5] }\end{array}$ \\
\hline Simple & $\begin{array}{l}\text { Flight based on compass orientation at launch. } \\
\text { Pushing RC stick South will always fly in } \\
\text { South direction [6] }\end{array}$ \\
\hline Altitude Hold & $\begin{array}{l}\text { Stable mode with throttle automatically } \\
\text { maintained to keep the current altitude[7] }\end{array}$ \\
\hline
\end{tabular}


Figure 1: Quadcopters built for the Swarm-Copters System

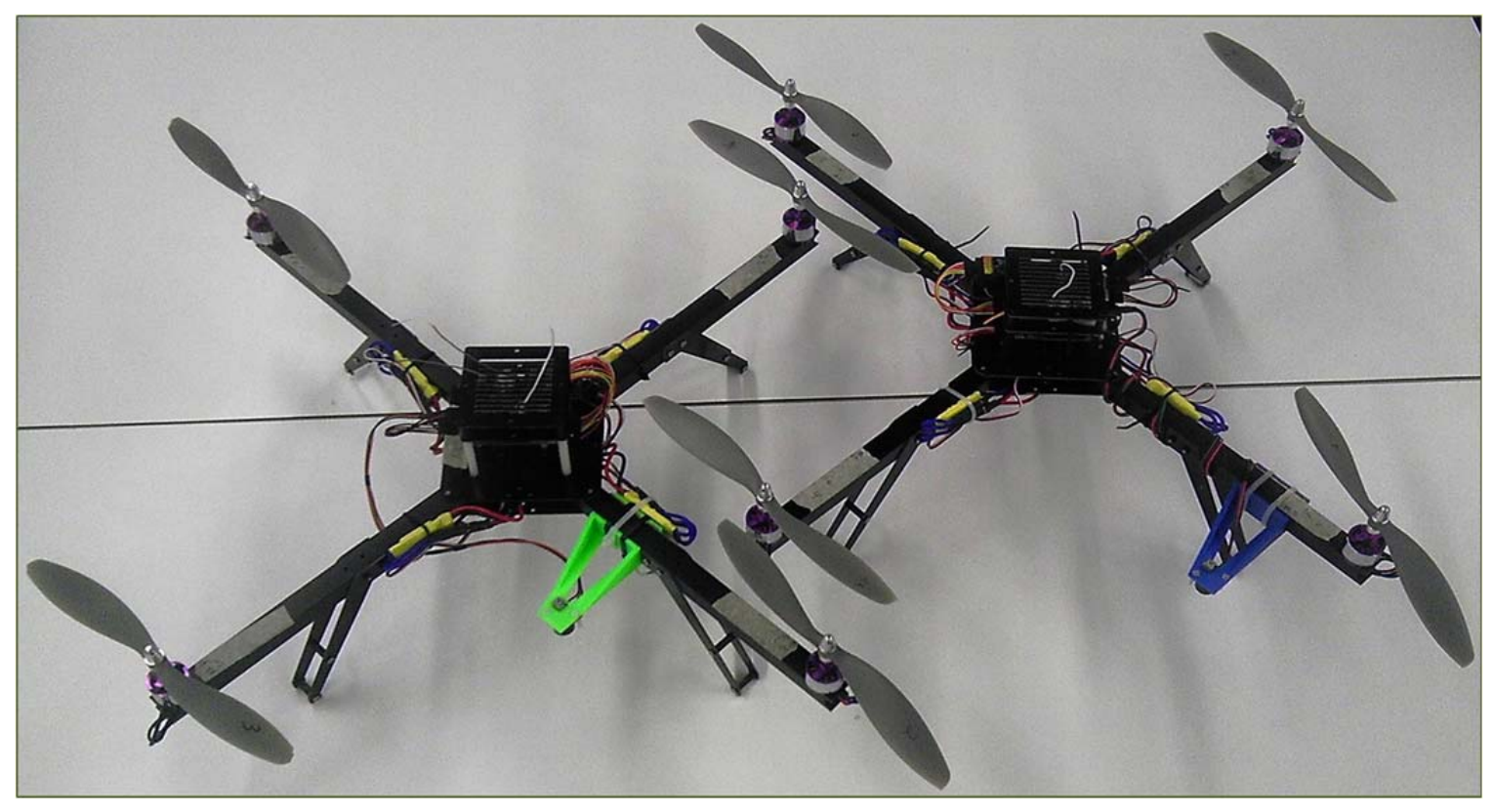


Figure 2: Screenshot of QGroundControl Mission Planner software running on the Base Station sub-system.

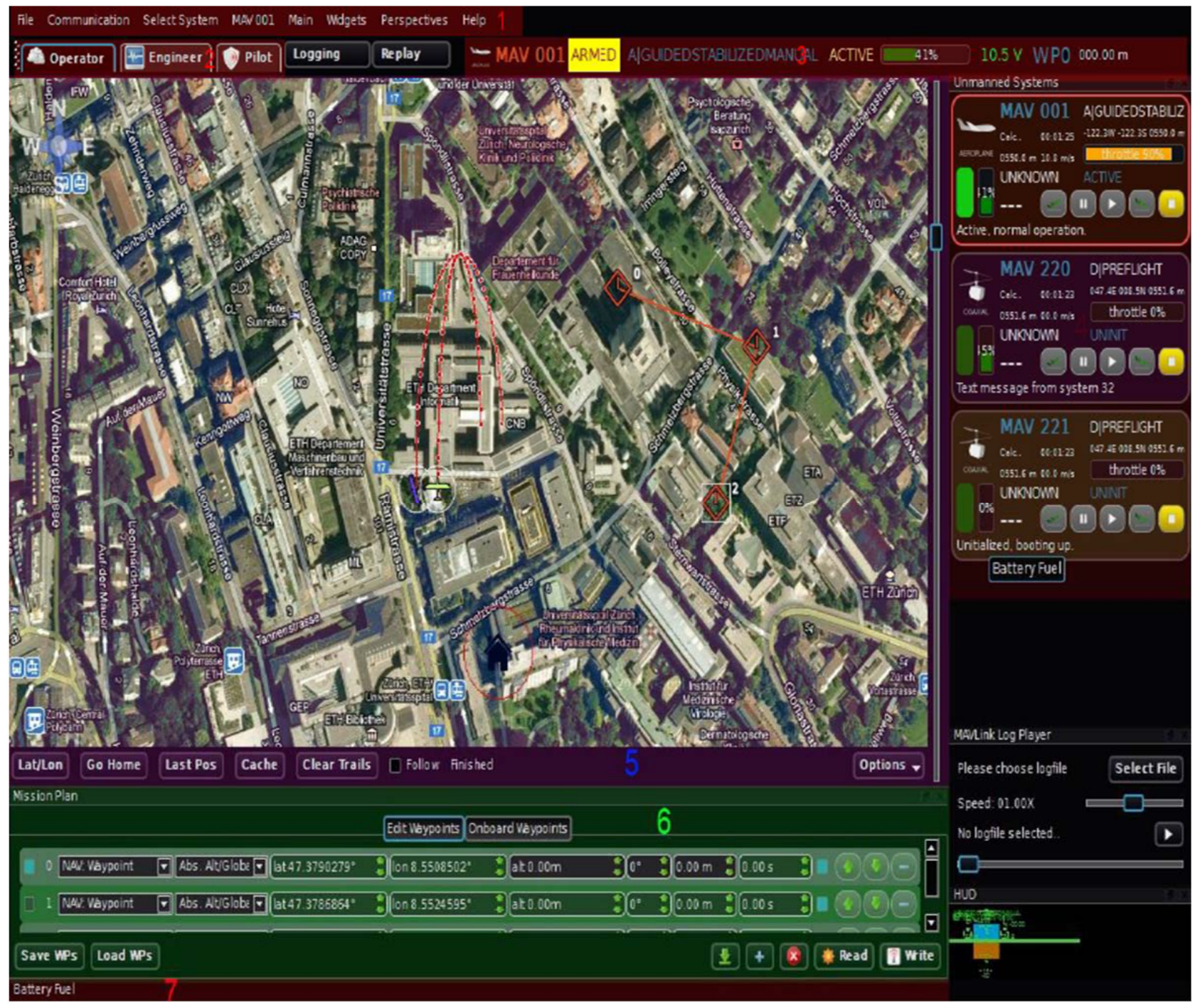


Figure 3: Sub-System Organization and Relationships

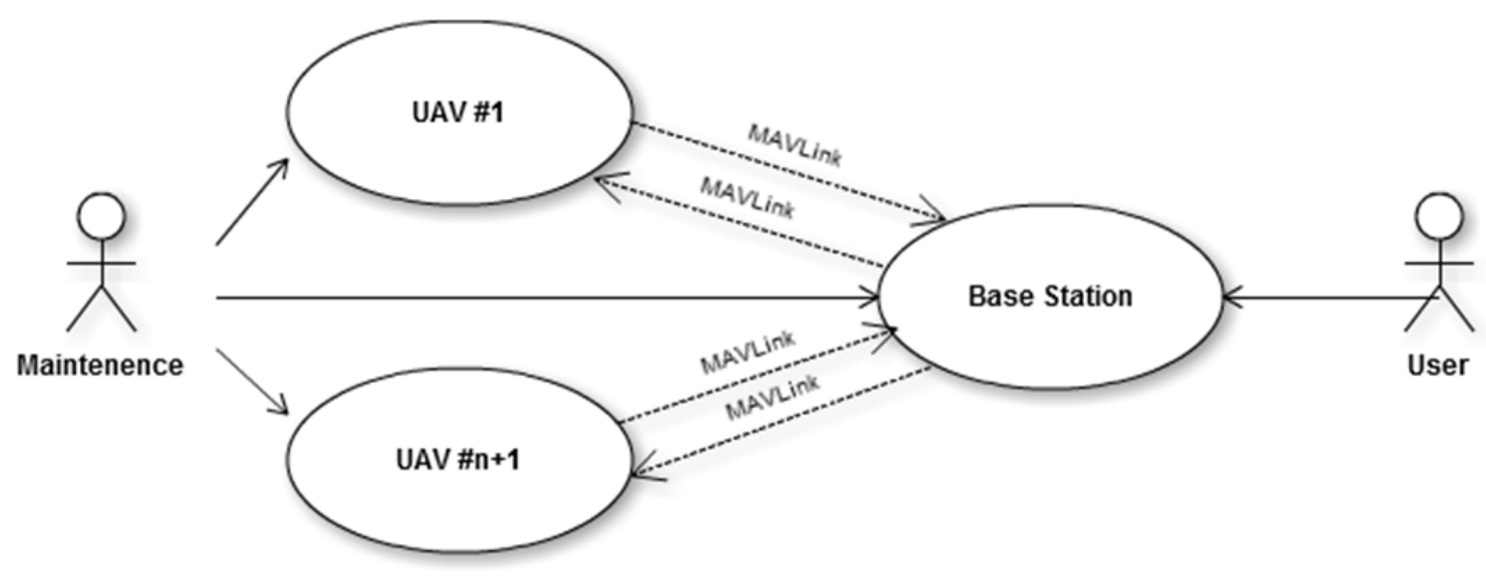

